\title{
Designing 2D arrays for SHM of planar structures: a review
}

\author{
Tadeusz Stepinski ${ }^{a, b}$, Łukasz Ambrozinski $^{a}$, and Tadeusz Uhl ${ }^{a}$ \\ ${ }^{a}$ AGH University of Science and Technology, al. Mickiewicza 30, Krakow, Poland; \\ ${ }^{b}$ Uppsala University, Uppsala, Sweden
}

\begin{abstract}
Monitoring structural integrity of large planar structures that aims at detecting and localizing impact or damage at any point of the structure requires normally a relatively dense network of uniformly distributed ultrasonic sensors. 2-D ultrasonic phased arrays, due to their beam-steering capability and all azimuth angle coverage are a very promising tool for structural health monitoring (SHM) of plate-like structures using Lamb waves (LW). Linear phased arrays that have been proposed for that purpose, produce mirrored image characterized by azimuth dependent resolution, which prevents unequivocal damage localization. $2 \mathrm{D}$ arrays do not have this drawback and they are even capable of mode selectivity when generating and receiving LWs. Performance of 2D arrays depends on their topology as well as the number of elements (transducers) used and their spacing in terms of wavelength.

In this paper we propose a consistent methodology for three-step: theoretical, numerical and experimental investigation of a diversity of 2D array topologies in SHM applications. In the first step, the theoretical evaluation is performed using frequency-dependent structure transfer function (STF). STF that defines linear propagation of different LWs modes through the dispersive medium enables theoretical investigation of the particular array performance for a predefined tone-burst excitation signal.
\end{abstract}

A dedicated software tool has been developed for the numerical evaluation of $2 \mathrm{D}$ array directional characteristics (beampattern) in a specific structure. The simulations are performed using local interaction simulation approach (LISA), implemented using NVIDIA CUDA graphical computation unit (GPU), which enables timeefficient 3D simulations of LWs propagation.

Beampatterns of a 2D array can be to some extend evaluated analytically and using numerical simulations; in most cases, however, they require experimental verification. Using scanning laser vibrometer is proposed for that purpose, in a setup where LWs, excited by PZT transmitters of the investigated array are sensed in multiple points corresponding to the locations of the $2 \mathrm{D}$ array receiving elements. A virtual receiving sub-array is created in this way and the performance of various array architectures in the reception mode can be evaluated experimentally without the need of physical prototype; a change of topology requires only straightforward modification of the measurement points distribution at the tested structure.

For illustration, beampatterns of three symmetrical 2D topologies, i.e., circular, star-shaped and spiralshaped, will be examined in the paper and compared in terms of their beam-width and side-lobes level. The effect of apodization applied to the array elements will be also investigated.

Keywords: Lamb waves, Phased Array, SHM.

\section{INTRODUCTION}

Structural health monitoring (SHM) of plate-like structures is a hot topic of research since many of such constructions must meet high safety standards. Lamb waves (LW) are a promising tool for these applications since thanks to their ability to propagate over long distances and sensitivity to various types of damages. ${ }^{1}$ A critical factor for SHM of plate-like structures is the design of transducers and its distribution over the investigated plate. A well known approach in this field is the use of transducers distributed over a wide area of the inspected structure. $^{2}$

tstepin@agh.edu.pl, ambrozin@agh.edu.pl, tuhl@agh.edu.pl

Nondestructive Characterization for Composite Materials, Aerospace Engineering, Civil Infrastructure, and Homeland Security 2013, edited by Tzu Yang Yu, Andrew L. Gyekenyesi, Peter J. Shull, Aaron A. Diaz, H. Felix Wu, Proc. of SPIE Vol. 8694, 86941R - @ 2013 SPIE · CCC code: 0277-786X/13/\$18 · doi: 10.1117/12.2010000 
Another alternative is using phased arrays (PA) with elements located closely to each other. Performance of a SHM system can be enhanced by means of active ultrasonic PAs due to their superior signal to noise-ratio and beam-steering capability. ${ }^{3}$ The concept of beam-steering of ultrasonic waves with the use of a PA is wellestablished both in medical ${ }^{4}$ and $\mathrm{NDT}^{5}$ imaging. It appeared, however, that bringing the method for SHM of plate-like structures requires arrays with 2D topologies to avoid equivocal damage localization. ${ }^{3}$ Therefore, a number of reports has been published concerning various shapes of $2 \mathrm{D}$ arrays, for instance, circular, $^{6}$ square, ${ }^{7}$ spiral $^{8}$ and $\operatorname{star}^{9}$ shaped arrays.

Quality of an image created with the use of an array of a defined shape depends also, among other factors, on the array's aperture and imaging technique used. Moving sensors can be used in sonar and mechanized NDT applications to increase array's aperture by means of synthetic aperture focusing technique (SAFT). ${ }^{10}$ Since this solution would be infeasible in most SHM applications, this paper is concerned with the static arrays that are permanently attached to the monitored structure and used in SHM applications. These arrays can take advantage of multiple emitting transducers capable of illuminating damage from a set of diverse localizations, which results in an extension of array's effective aperture. ${ }^{11}$

Another critical factor that affects performance of a PAs-based SHM system is signal processing technique implemented in the beamforming scheme. The most common scheme used, mostly due to its simplicity and robustness for processing snapshots captured by an array, employs delay and sum (DAS) operations in time domain. More advanced methods capable of angular ${ }^{12}$ and radial resolution ${ }^{13,14}$ improvement can also be implemented to Lamb waves sensed using PAs. Advanced processing techniques, however, require precise information on material properties, which can create limitations in practical applications, especially, to anisotropic materials.

\section{THEORETICAL BACKGROUND}

Application of array technique to SHM of plate-like structures involves a set of transducers that are permanently attached to, or embedded into the structure to enable performing inspection of a large region of interest (ROI) from a fixed location. In an active pulse-echo setup the transmitting elements of the array generate elastic waves in the plate that are scattered from the discontinuities present in the structure and the scattered waves are subsequently sensed by the array's receiving elements.

If an array is used in the phased array (PA) mode, the transmitting and receiving sub-arrays consist normally of the same elements. In transmission the elements are excited by time-shifted pulses to obtain a steered wavefront, which is received by the same elements in the reception mode and amplified by introducing respective time delays bringing the received pulses in phase. Beamformers implementing this technique, referred to as delay-and-sum (DAS), are commonly used in ultrasound applications. Using steered and possibly focused beam improves angular (azimuth) resolution in ultrasonic image but it requires scanning of the whole ROI. This means that the send-receive cycle has to be repeated for a number of azimuths and ranges within the ROI, which results in a low acquisition speed.

An alternative to PA is the synthetic aperture (SA) approach that has been developed for radar (SAR synthetic aperture radar) and more recently applied in medical ultrasound. Generally, SA beamforming uses different transmitting and receiving sub-arrays for imaging, which is referred to as multistatic mode. In classical SA technique image is formed by successive excitation of all transmiting elements and reception of the scattered waves by all other array elements. The final image is formed as a superposition of beamformed energy from all transmitted cycles. A SA image is generated in result of post-processing of all received data which requires rather intensive computations. A SA image is essentially equivalent to an image produced by conventional PA using a number of transmit-receive focused beam cycles equal to the number of pixels in the ROI.

For a linear array with $N$ elements a full matrix of $N^{2}$ transmit-receive data can be acquired if all the elements are fired in $N$ transmission cycles. There are, however, many other ways of gathering data for SA processing that depend on the array geometry and topology of the transmitting aperture. Most of these ways are designed to reduced number of transmissions with minor loss of resolution and an acceptable signal to noise ratio. Generally, this can be achieved by using sparse transmitting apertures. If elements of a sparse transmitting aperture are distributed across the full aperture of the array, there will be no loss in the field of view and only a minor loss in lateral resolution. 


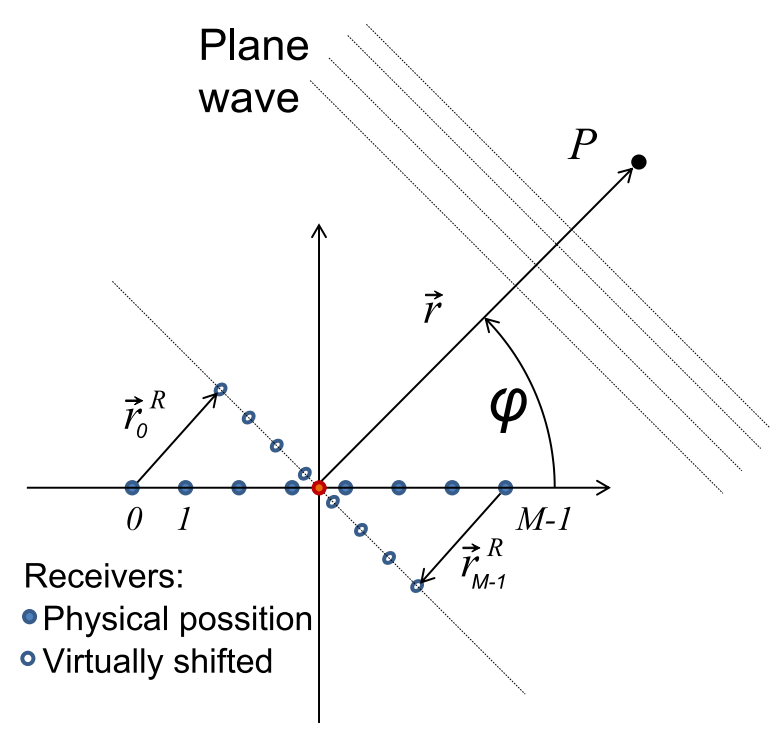

(a)

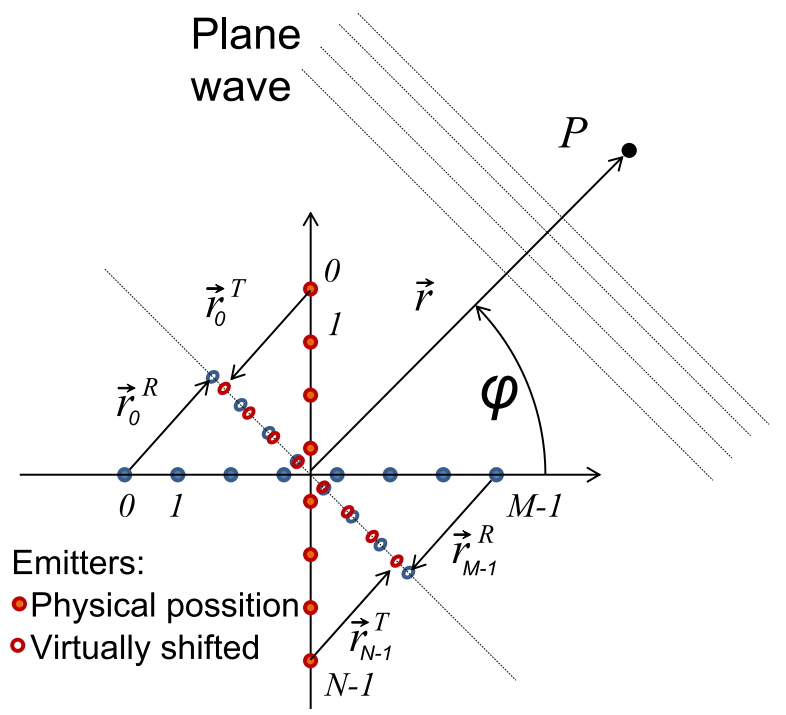

(b)

Figure 1. Illustration of imaging under far-field assumption a single emitter and uniform linear sensing array (a), synthetic aperture using a linear array of emitters and linear array of sensors (b).

\subsection{Imaging in the monostatic mode}

Consider a setup consisting of an uniform linear sensing array of $M$ transducers, presented in fig. 1a, and a point-like transmitter localized in point $(0,0)$. The transmitter is an omni-directional point-like source of a single dispersive Lamb mode. Due to the dispersive character, the individual waves travel at a different velocity than the wave packet. Velocity of the individual waves is determined by phase velocity, which directly affects the phase of the wave. Group velocity, related to the speed of the group of individual packets, determines the velocity of wave energy transportation. ${ }^{3}$

Assume now a point-like scatterer $P$ in the array's far-field at the distance $|\vec{r}|$ and azimuth $\phi$. The excited wave propagates through the medium and when it hits the target it is scattered and propagates towards the sensing array. The time-shift between the excited wave and the reflected wave-packet, which reached the point $(0,0)$ is simply

$$
\tau_{g}(|\vec{r}|)=\frac{2|\vec{r}|}{c_{g}}
$$

where $c_{g}$ denotes group velocity of the LW.

If the scatterer is in the array's far-field, the reflected wave can be assumed to be planar and its wavefront in $(0,0)$ forms a line perpendicular to the direction of arrival. Since the distances between the subsequent receivers and the wavefront are different the captured snapshots will be shifted in phase. Under the assumption specified above, the time-shifts due to wave propagation can be easily calculated for $m$ th array element using the following trigonometric expression

$$
\tau_{m}(\phi)=\frac{d\left(m-\frac{M-1}{2}\right) \cos \phi}{c_{p}}
$$

where $c_{p}$ is the phase velocity of the wave, and $d$ is sensor spacing.

The beamforming algorithm compensates for these time-shifts when the array is steered in the direction of wave arrival. The compensation can be considered as a virtual shift of the emitting/receiving elements. ${ }^{6}$

In the approach presented here, imaging is performed in the form of a bitmap in a polar coordinate system where each pixel can be defined with its angular position $\alpha$ and the distance from the center $|\vec{r}|$. Then, equation, 
which defines pixel intensity can be written as follows

$$
I(|\vec{r}|, \alpha)=\sum_{m=0}^{M-1} w_{m} y_{m}\left(\tau_{m}(\alpha)+\tau_{g}(|\vec{r}|)\right),
$$

where $y_{m}$ is the temporal signal captured by the $m$ th sensor contributing to imaging with an apodization weight $w_{m}$.

To localize the scatterer, the image has to be created for a set of azimuths. If $\alpha$ equals to the angle of incidence $\phi$ the time-delays are compensated, which results in the alignment of the snapshots shown schematically in fig. 1a. Thus, the aligned signals will be added coherently which results in the enhanced contrast of the resulting image.

\subsection{Imaging in the multistatic mode}

Assume now that an array of $M$ sensing an $N$ emitting elements is used for imaging, as presented in fig. 1b. In the multistatic SA imaging mode the transmitters are subsequently fired and the responses are captured by the sensing elements. Damage imaging can be performed similarly to the previous case, however, the time shifts for the emitting elements should be also compensated. A separate image is produced for each emission-reception cycle and the final result is formed as a sum of those images. Then, intensity of the pixel $I(|\vec{r}|, \alpha)$ can be evaluated as

$$
I(|\vec{r}|, \alpha)=\sum_{n=0}^{N-1} \sum_{m=0}^{M-1} w_{T}^{n} w_{R}^{m} y_{m, n}\left(\tau_{m}^{R}(\alpha)+\tau_{n}^{T}(\alpha)+\tau_{g}(|\vec{r}|)\right),
$$

where the terms $\tau_{n}^{T}(\alpha)$ and $\tau_{m}^{R}(\alpha)$ denote the time-delays required to steer the transmitting and receiving subarray, respectively, with the angle $\alpha$, and $y_{m, n}$ is the temporal signal captured by the $m$ th sensor due to the excitation by the $n$th transmitter.

If the hardware used for imaging is capable of simultaneous excitation of the time-shifted signals from the emitting elements, the transmitting array can be used to steer the wave at a predefined set of azimuths in a sequence of transmissions. Response to each transmitted wavefront is captured by the receiving elements and since the wave steering is already done in the transmission the expression for imaging simplifies to

$$
I(|\vec{r}|, \alpha)=\sum_{m=0}^{M-1} y_{m, \alpha_{T}}\left(\tau_{m}(\alpha)+\tau_{g}(|\vec{r}|)\right),
$$

where $y_{m, \alpha_{T}}$ is the response of the $m$ th sensor due to steered transmission of the wavefront at the azimuth $\alpha_{T}$.

If imaging is performed with an angular resolution of the transmission azimuths $\Delta \alpha$, the angle $\alpha$ in the eq. (5) can be replaced by $\alpha_{T}=m \Delta \alpha+\alpha_{0}$. In general case, however, imaging can be performed with different resolution steps in transmission and reception. In such a case the data obtained for the transmission angle $\alpha_{T}$ is used to generate the image at a larger set of angles $\alpha$, e.g., defined by the relation $\left|\alpha_{T}-\alpha\right| \leq \Delta \alpha / 2$.

\section{SIMULATIONS}

The evaluation of beampattern assumes normally a monochromatic, continuous wave excitation. In SHM applications, however, tone-burst or broad-band pulses are used to enable damage localization by the time of flight extraction of the damage-related reflections. Since the shape of the excitation signal plays a vital role in the mechanism of Lamb wave propagation and their beamforming, in this section simulations of Lamb wave propagation for a broadband excitation signal will be presented. The responses processed with beamforming algorithms will be used to present and discuss performance of the selected 2D arrays.

Lamb waves are dispersive and multimodal. Relation between the phase velocity and the product of the plate thickness and excitation frequency can be evaluated by numerical solution of Rayleigh-Lamb equation. When the dispersion curves are available it is possible to evaluate the response of the structure due to the defined excitation using frequency-dependent STF. ${ }^{15}$ 
This method for evaluation of dispersive signals was used to simulate wave propagation in $2 \mathrm{~mm}$ thick aluminum plate. In the presented approach a set of point-like transducers arranged in regular 2D arrays, acting as transmitters or receivers, was assumed. Moreover, a point-like scatterer localized in far-field of the array was assumed. In the simulations of wave propagation, based on the STF approach, the length of the propagation path was equal to the transmitter-scatterer-receiver distance. In order to pay more attention to the dispersion phenomenon than to the multimode nature of the LWs, a single mode excitation was assumed.

\subsection{Monostatic imaging}

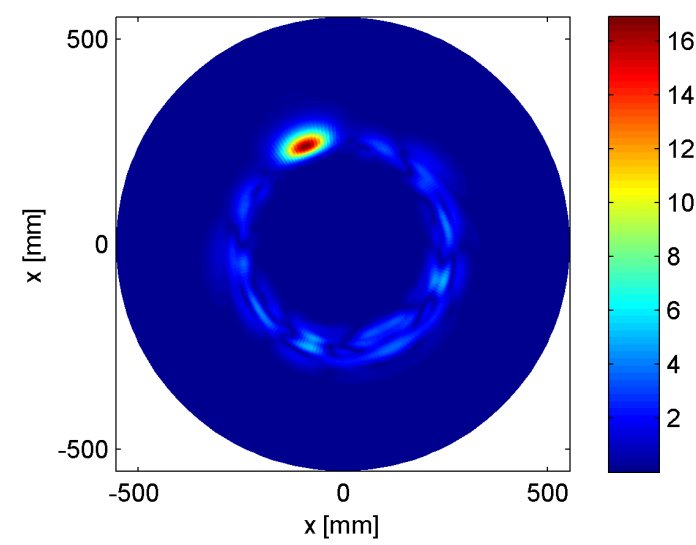

(a)

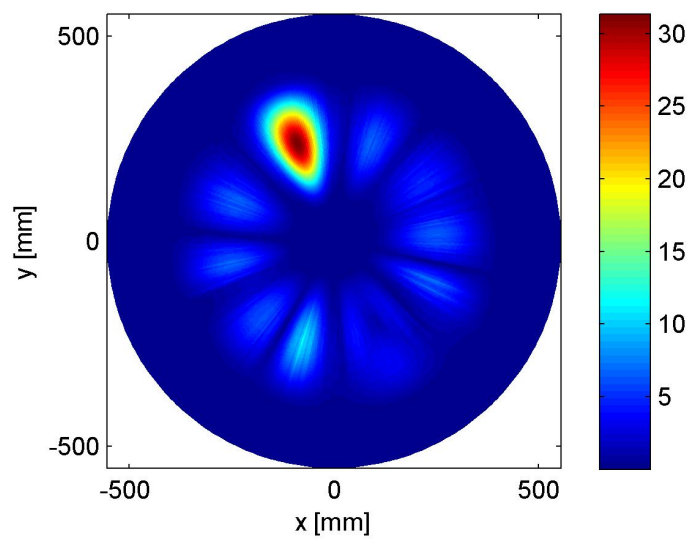

(b)

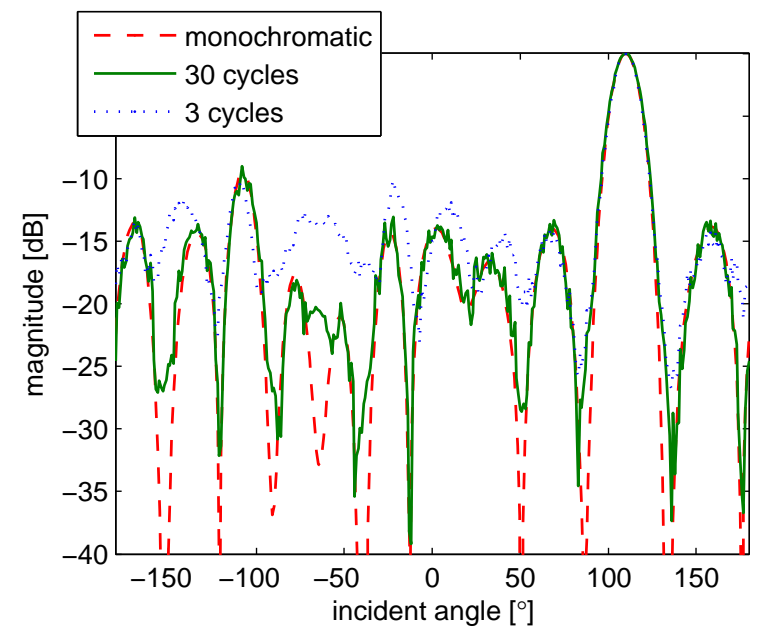

(c)

Figure 2. Target images obtained in the starlike STMR setup using STF for the single $A_{0}$ mode excited with windowed tone burst consisting of $3 \mathrm{a}$ ), and 30 sine cycles b). Beampatterns obtained for the tone-burst excitation signals and for the monochromatic excitation c).

The first simulation series was concerned with the monostatic setup consisting of a single transmitter and multiple receivers (STMR) array. The receiving elements were distributed according to the topology of starshaped array, presented in fig. 3a, whereas the transmitting element was placed in the center of the array. A point-like scatterer was localized at a distance of $250 \mathrm{~mm}$ from the array center at an azimuth $110^{\circ}$.

Two simulations were conducted for this setup: first, a tone-burst signal consisting of 3 cycles of sine at a frequency of $100 \mathrm{kHz}$, modulated with Hanning window was used as an excitation. In the second simulation the number of cycles was increased to 30. The spacing of array's elements was equal to half of wavelength of 
the $A_{0}$ mode at the excitation frequency. The responses captured by the consecutive sensors were evaluated using STF approach. Both sets of the simulated snapshots were processed with the DAS beamformer assuming far-field approximation; the resulting images are shown in fig. 2a and b. It can be seen that the longer burst signal results in poor range resolution. The beampatterns obtained from the maximum amplitudes of the sum of the beamformed signals in function of azimuth are presented in fig. 2c where they can be compared with the theoretical directivity characteristics of the same array implemented for a $2 \mathrm{D}$ case, assuming infinite, monochromatic exciting signal. Good agreement of the compared beampatterns can be observed, however, the response obtained for the 30 cycle excitation is closer to the monochromatic theoretical beampattern since it has deeper minima due to the destructive interference.

\subsubsection{Influence of array's shape}

In order to illustrate the influence of array topology on beamforming, various topologies presented in fig. 3 will be discussed below. All analyzed arrays will consist of 32 sensors. The star-shaped array, presented in fig. 3a consists of 4 linear sub-arrays intersecting at an angle of $45^{\circ}$. The elements of the sub-arrays are spaced at a distance $d=5 \mathrm{~mm}$, therefore the outside diameter of the array was $40 \mathrm{~mm}$. The circular-shaped topology shown in fig. 3b was made up of 2 concentric circles. The diameter of the larger one was $25.62 \mathrm{~mm}$ and the distance denoted $d$ in the figure is equal to $5 \mathrm{~mm}$. Finally, the spiral-shaped array, presented in fig. 3c was created in the way described in. ${ }^{8}$ The transducers are placed on concentric circles with radii increasing with a step of $5 \mathrm{~mm}$, 4 sensors are placed in each circle, which is rotated with an angle of $15^{\circ}$ with respect to the former one. The spacing of the subsequent sensors varied form 5.11 to $8.87 \mathrm{~mm}$ and the outside diameter of the array is $80 \mathrm{~mm}$.

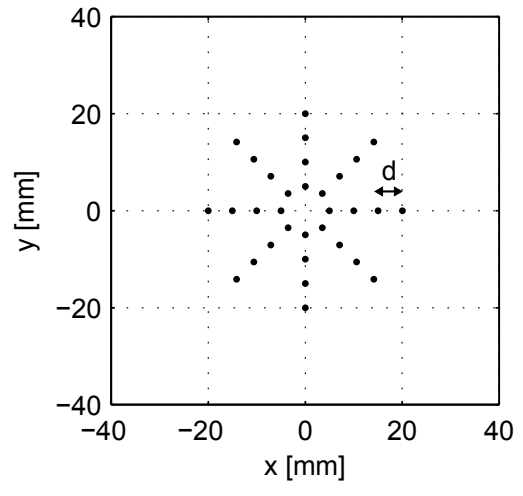

(a)

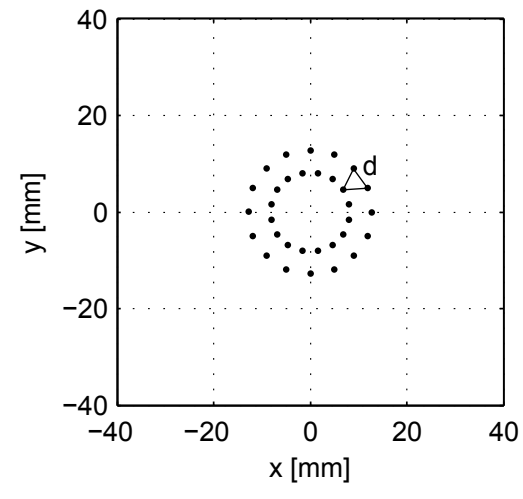

(b)

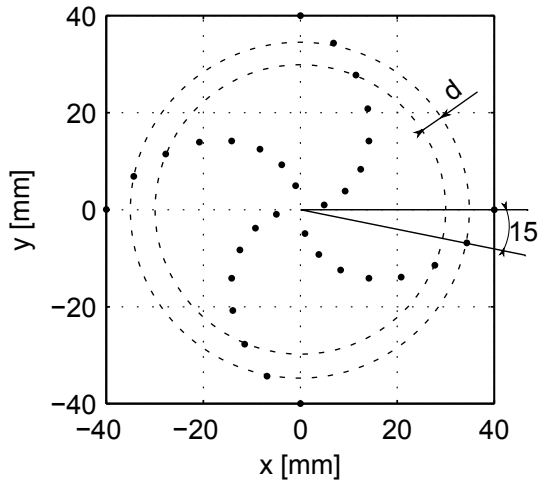

(c)

Figure 3. Investigated topologies of the array: (a) star-shaped, (b) circular, (c) spiral.

All of the investigated topologies have at least 2 axis of symmetry, therefore evaluation of the beampatterns can be limited to the range of $0^{\circ}-90^{\circ}$ and mirrored for the remaining directions.

The methodology presented in our previous work ${ }^{16}$ was used to process the theoretical, numerical and experimental signals. An example of beampatterns obtained using this procedure can be seen in fig. 4a, b, c for the wave with an arbitrary selected incident angle of $60^{\circ}$, for spiral, circular and star-shaped array, respectively. Good agreement of the directivity characteristics obtained theoretically, numerically and experimentally can be observed for all of the topologies, however, the experimental beampatterns are better reproduced by the simulated than the theoretical ones. It can be explained by the fact that LISA is a more accurate method for modeling of Lamb waves propagation than the theoretical approach based on the simplified STF model.

The beampatterns obtained for the waves with incident angle in the range of $0^{\circ}-90^{\circ}$ were analyzed and selected parameters estimated from the characteristics were presented in our previous paper. ${ }^{16}$ Width of the main lobe was estimated as its angular width at the level of $-3 \mathrm{~dB}$. The maximal and minimal values of lobe width as well as the highest and the lowest sidelobe levels are investigated. From the results it could be observed that the spiral array has the narrowest main lobe among the investigated topologies, which was expected since 
this array has the largest aperture, however it the spiral-shaped array presented a significant side-lobes level. The circular array presented almost constant properties for different angles, however the performance was poor due to small aperture. Therefore a star-shaped array, as a compromise between the performance and simplicity was selected for the further analysis.

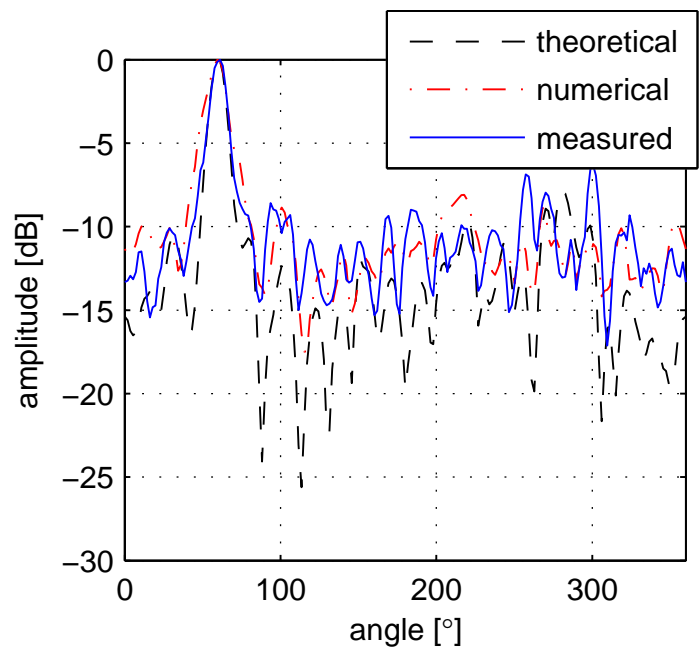

(a)

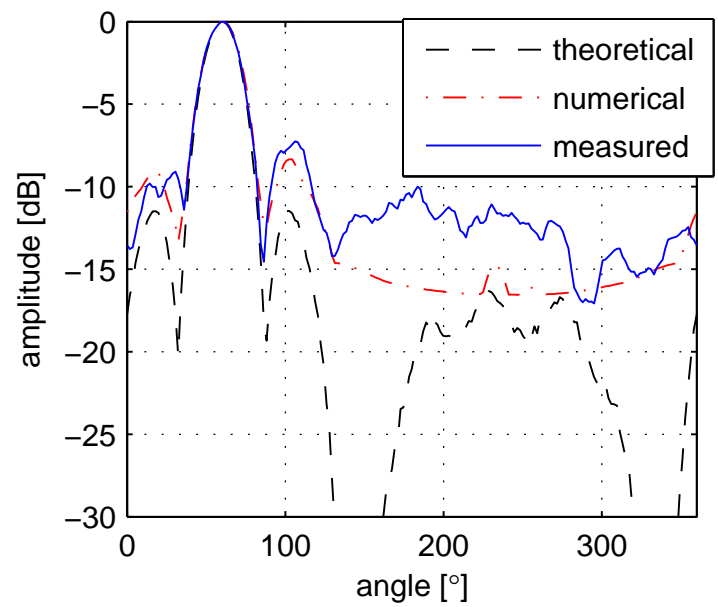

(b)

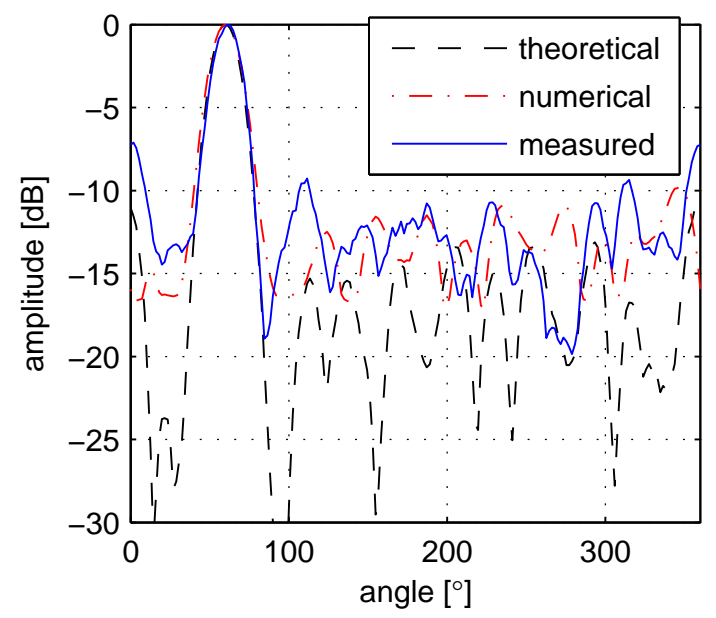

(c)

Figure 4. Beampatterns evaluated for the wave with incident angle $60^{\circ}$ for: (a) spiral, (b) circular and (c) star-shaped array. The theoretical profiles were obtained using the STF approach while the numerical ones by means of the numerical LISA simulation.

\subsection{Multistatic imaging}

In the next simulation the SA concept was illustrated in the multistatic setup consisting of multiple transmitters and multiple receivers (MTMR-SA) implemented in the form of star-shaped array of transmitters/receivers presented in fig. $7 \mathrm{~d}$. A point-like scatterer was localized at a distance of $250 \mathrm{~mm}$ at an angle of $110^{\circ}$ from the array. The simulated responses from the each transmitter-receiver pair formed a matrix consisting $8 \times 24$ time-traces. The snapshots were then processed with the DAS algorithm and the resulting image obtained using eq. (4) is presented in fig. 5a. If this image is compared to that in $2 \mathrm{a}$ it can be easily seen that, although the same topology of the array and the same number of elements was used, considerably improved result can 


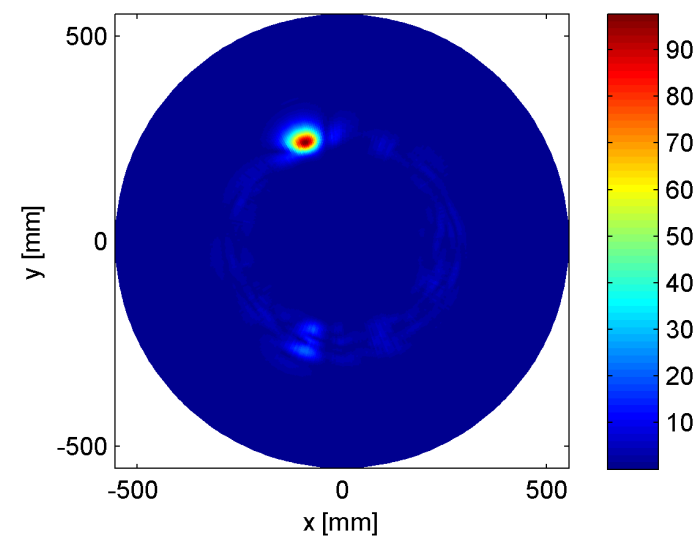

(a)

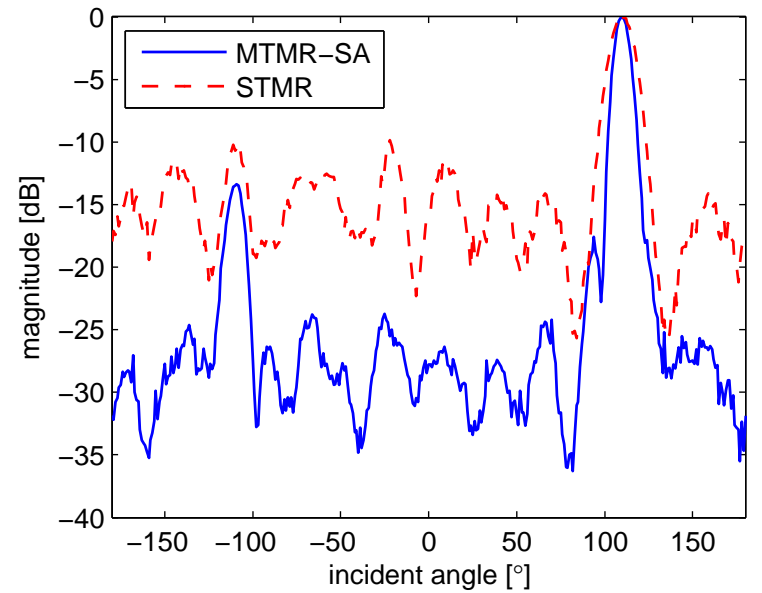

(b)

Figure 5. Target image obtained using the synthetic aperture MTMR-SA concept in the starlike setup a). Comparison of the resulting beampattern with that obtained with the STMR (fig. 2a), b). The profiles were obtained using STF.

be obtained this way. Both images were processed to find maxima occurring at the successive azimuths and their beampatterns were presented in fig. 5b. Comparison of the results shows that the multistatic approach is superior to the monostatic one in terms of main lobe width and range resolution.

\subsection{Imaging using an active transmitting array}

In the next series of the MTMR simulations the star shaped array, presented in fig. 3a and in more detail in $7 \mathrm{~d}$, was also used in the sweeping PA mode. In this mode the elements of the horizontal transmitting array were fired using time-shifted signals to form a plane wave sweeping the ROI. The backscattered signals were received by the star arms and post-processed to obtain high resolution image. In other words, focusing in the transmission was done in the material and focusing in the reception was performed off-line by the DAS operation of the captured snapshots.

In the presented simulation, azimuth of a far-field point-like scatterer was, like previously, $110^{\circ}$. Since the transmitting array was linear and it produced a mirrored lobe, the transmission angle was limited to the range $0-180^{\circ}$ but various angular sweeping steps were considered. In the first step the beam was transmitted at a set of azimuths with the step of $10^{\circ}$, which lead to the 19x24 matrix of time-traces (19 azimuths and 24 receiving elements). The same resolution was used to process the captured snapshots, the result can be seen in fig. 5a.

Next, the same data was processed with $1^{\circ}$ angular resolution in the reception which yielded the result can be seen in fig. 6b. Finally, the simulation of sweeping with $1^{\circ}$ resolution both in the transmission and reception was performed resulting in 181x24 matrix of time-traces (181 azimuths and 24 receiving elements); the results are presented in fig. 6c. Comparison of the images shown in fig. 6a, b and c shows that rather poor resolution obtained for the $10^{\circ}$ step cannot be improved if the captured data is processed with a higher resolution in the reception and smaller step in the transmission is required for that.

The beampatterns obtained for the images from fig. $6 \mathrm{c}$ and fig. 2a can be compared in fig. $6 \mathrm{~d}$. From the plots it can be seen that both approaches, the synthetic MTMR-SA focusing and the MTMR-PA sweeping, yield the same result.

\section{EXPERIMENTS}

The experiment results presented in this section illustrate imaging with the use of MTMR. In these experiments a linear array of PZTs was used for multistatic and PA transmission, whereas the the measurement of the responses was conducted in a contactless manner by means of a laser scanning Doppler vibrometer (LSDV). 


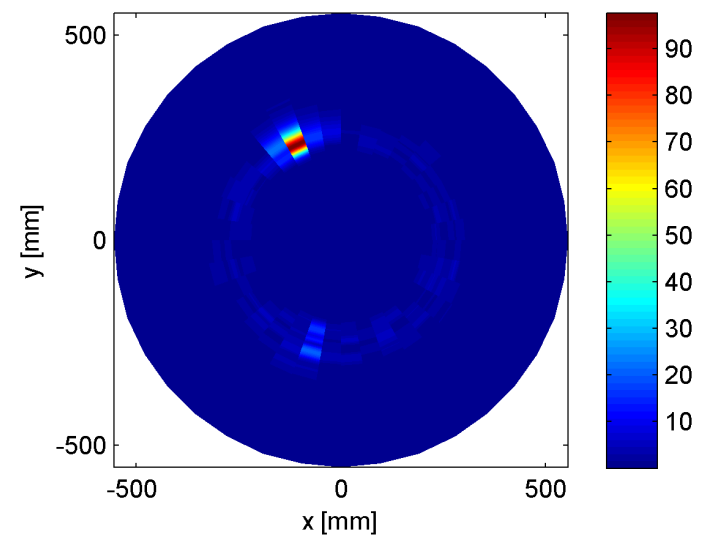

(a)

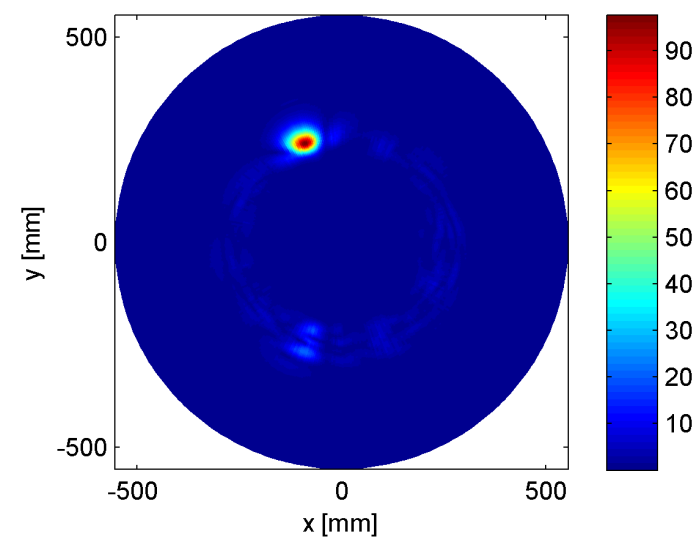

(c)

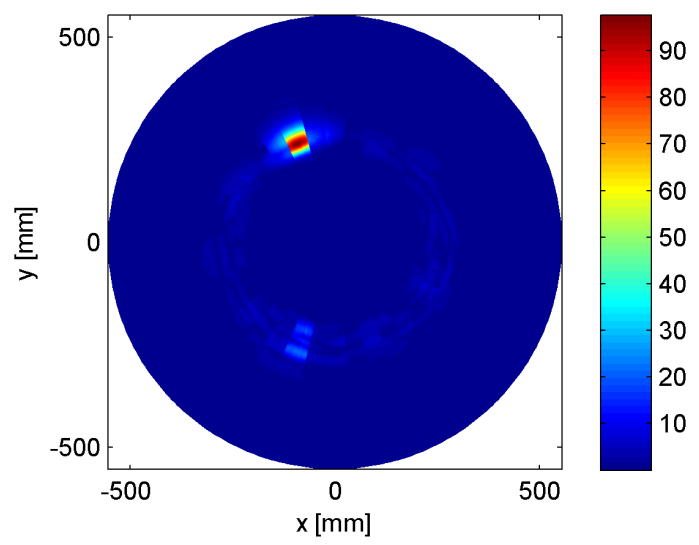

(b)

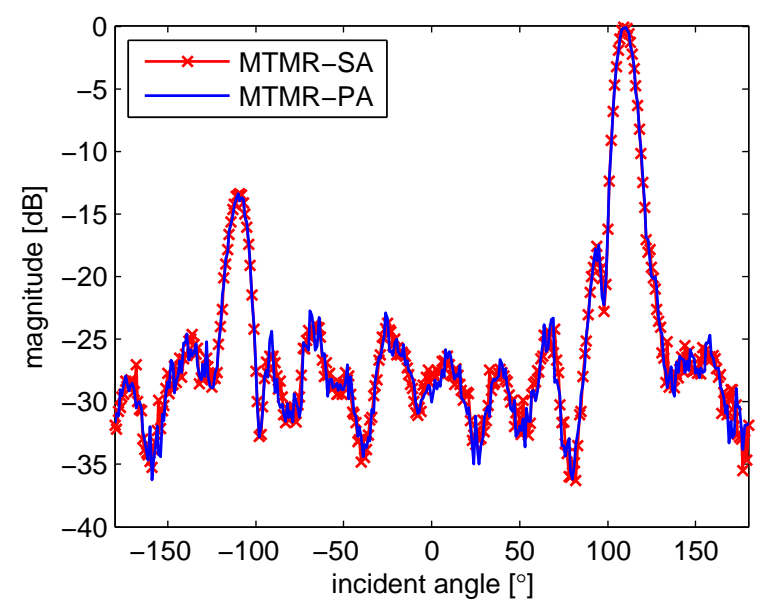

(d)

Figure 6. Target images obtained in the STF simulations of the MTMR-PA mode for the following emission/reception sweeping steps: $\left.\left.10^{\circ} / 10^{\circ} \mathrm{a}\right), 10^{\circ} / 1^{\circ} \mathrm{b}\right), 1^{\circ} / 1^{\circ} \mathrm{c}$ ). The beampatterns obtained using the MTMR-SA and the MTMR-PA mode with azimuth sweeping step $1^{\circ} / 1^{\circ} \mathrm{d}$ ).

\subsection{Multistatic imaging}

The experiments were conducted on an aluminum plate of the size 1000x1000x2 mm. An uniform linear array of transmitters was mounted at the center of the plate. The array consisted of $82 \times 2 \times 2 \mathrm{~mm}$ PZT elements, type CMAP 12 from Noliac Denmark, spaced at a distance of $5 \mathrm{~mm}$. The responses of the structure were captured on the other side of the plate using laser scanning Doppler vibrometer. Tone-burst signals consisting of 3 cycles of sine modulated by Hanning window were used as an excitation. PAS- 8000 from EC Electronics, Poland, used as a signal generator, enabled both single-element excitation and simultaneous generation of the time-shifted signals in PA mode. The laser scanning points were set according to the sensors location presented in fig. $7 \mathrm{~d}$.

Great advantage of the contact-less measurement technique is that it facilitates modification of the measurement points grid which enables investigation of various topologies of the sensing sub-array. This setup has, however, a serious limitation - effects of the inter-element scattering that may be encountered for a full 2D array of PZT elements cannot be taken into account.

\subsubsection{Experimental evaluation of effective aperture}

The first experiment performed in this setup consisted in evaluating effective aperture of the array for an exemplary azimuth. A scatterer was placed in the array's far-field at an azimuth angle of $112^{\circ}$. In the first 


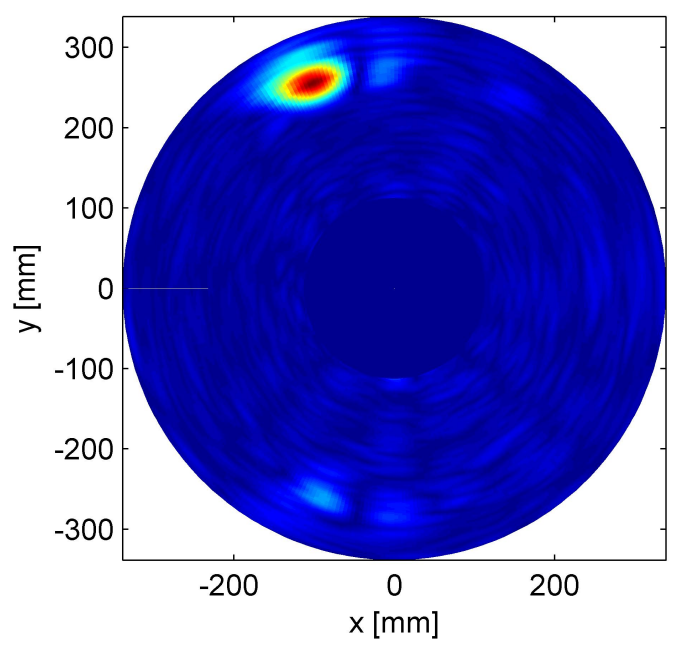

(a)

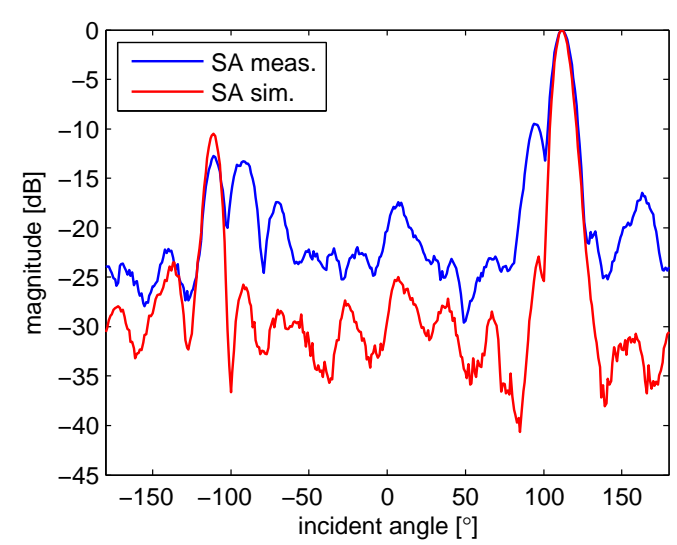

(c)

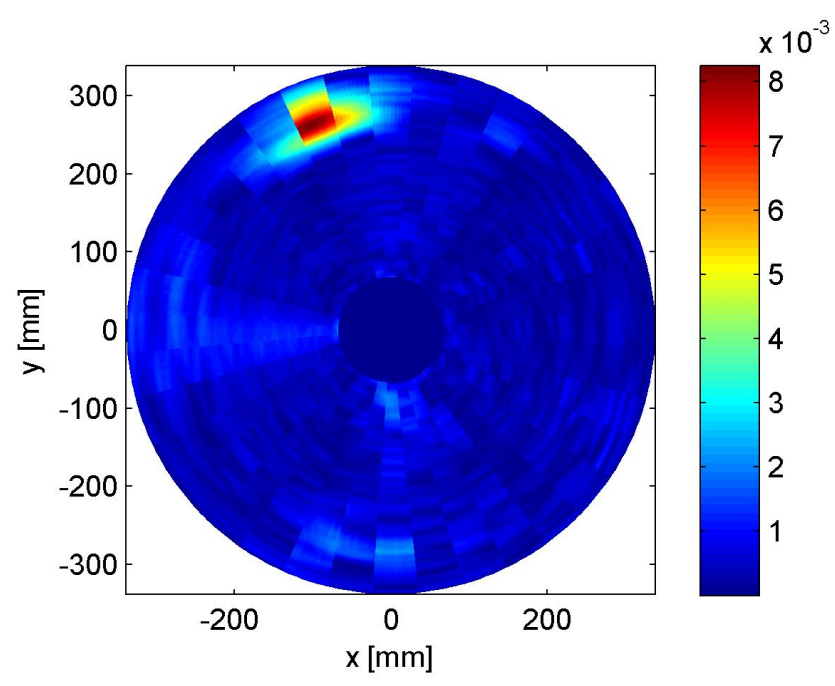

(b)

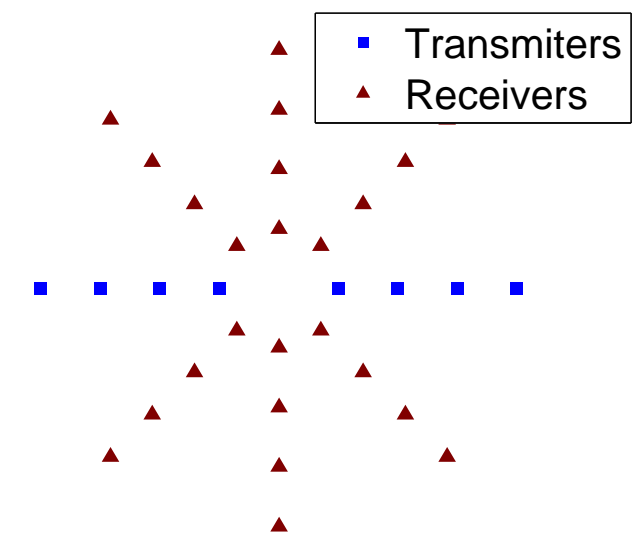

(d)

Figure 7. Experimental results of damage imaging with the use of the MTMR-SA a), the MTMR-PA excitation b); Comparison of the beampatterns obtained for the experimental and data simulated using STF c). Transmitters and receivers layout in the MTMR-SA experiment.

step, the MTMR-SA concept was used in which the subsequent elements were fired and the respective responses were captured. The snapshots were used for off-line imaging that yield the result shown in fig. 7a.

In the next experiment (the MTMR-PA) the elements of the transmitting array were excited simultaneously with time-shifted signals. The sweeping was performed for a set of angles in the range $0-180^{\circ}$ with a step of $10^{\circ}$. The acquired data was used for imaging with the resolution of $1^{\circ}$ and the results presented in fig $7 \mathrm{~b}$.

Comparing the results it can be seen that the MTMR-PA offers much worse resolution and lower image quality than the MTMR-SA, which is not surprising since the resolution in the active transmission step was low. Based on the image obtained with the use of SA a beampattern presented in $7 \mathrm{c}$ was obtained where it can be compared with the simulation result performed using the method STF described in sec. 3. Comparison of both beampatterns shows that the simulated and experimental results are in a good agreement in the term of mainlobe width, however, higher side-lobes levels and noise level can be observed for the experimental result. 


\subsubsection{Damage imaging with the use of synthetic aperture}

In the next experiment 3 scatterers were placed on the plate according to the setup resented in fig. 8a. The result of imaging performed with the MTMR-SA approach is shown in fig. 8b. From the image it can be observed that equivocal damage localization can be obtained with the use of SA and for each reflector a corresponding area of the image with high contrast exists.

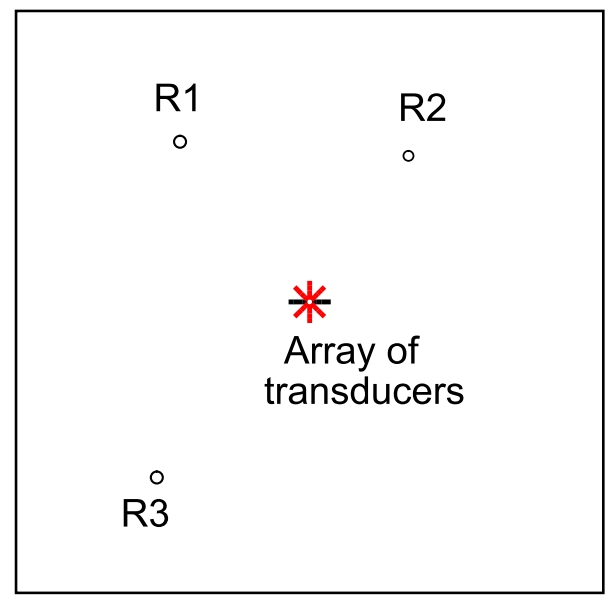

(a)

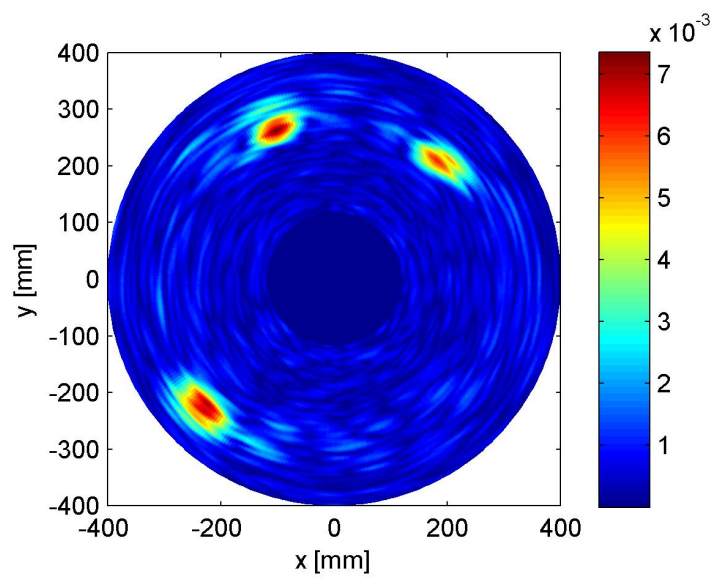

(b)

Figure 8. Multiple scattering experiment. Distribution of scatterers on the plate a), and results of imaging using of the MTMR-SA b).

\section{CONCLUSIONS}

Various approaches to beamforming of Lamb waves were presented in the paper; synthetic aperture technique was compared to the monostatic and self-focusing approach.

Based on the results, it can be concluded that the multistatic approach takes an advantage of multiple successive transmissions, which allows to illuminate targets from a set of diverse positions and results in a high quality of the produced image. Application of this technique enables obtaining narrower mainlobe and lower side-lobes level comparing to the monostatic setup using the same array topology.

It was shown in simulations that focusing can be performed partially in the inspected structure with the use of an active transmitting array operating in the PA mode. However, a large number of transmission-reception cycles is required to achieve high image resolution. Moreover, the active approach requires multiple channel system capable of simultaneous generation of time-shifted signals while in the multistatic setup a single, multiplexed output channel is sufficient. Therefore the synthetic aperture multistatic approach, in which the data can be acquired with by means of a relatively simple hardware and subsequently processed off-line with an arbitrary resolution, is superior to the active scheme in SHM applications.

\section{Acknowledgments}

Financial support acknowledged: the Polish Government grant N N503 141236 'Technology of the ultrasonic micro-transducers in Lamb wave based SHM'.

\section{REFERENCES}

1. A. Raghavan and C. E. S. Cesnik, "Review of guided-wave structural health monitoring," The Shock and Vibration Digest 39, p. 91114, March 2007.

2. J. E. Michaels, "Detection, localization and characterization of damage in plates with an in situ array of spatially distributed ultrasonic sensors," Smart Materials and Structures 17(3), p. 035035, 2008. 
3. V. Giurgiutiu, Structural Health Monitoring With Piezoelectric Wafer Active Sensors, Academic Press, New York, 2008.

4. M. Karaman, I. O. Wygant, O. Oralkan, and B. T. Khuri-Yakub, "Minimally redundant 2-D array designs for 3-D medical ultrasound imaging," IEEE Transactions on Medical Imaging 28, July 2009.

5. B. W. Drinkwater and P. D. Wilcox, "Ultrasonic arrays for non-destructive evaluation: A review," NDTEEE International 39(7), pp. $525-541,2006$.

6. P. Wilcox, "Omni-directional guided wave transducer arrays for the rapid inspection of large areas of plate structures," Ultrasonics, Ferroelectrics and Frequency Control, IEEE Transactions on 50, pp. 699 -709, june 2003.

7. M. Engholm and T. Stepinski, "Using 2-D arrays for sensing multimodal lamb waves," Proc. of SPIE 7649, 2010.

8. B. Yoo, A. S. Purekar, Y. Zhang, and D. J. Pines, "Piezoelectric-paint-based two-dimensional phased sensor arrays for structural health monitoring of thin panels," Smart Materials and Structures 19(7), p. 075017, 2010.

9. L. Ambrozinski, T. Stepinski, and T. Uhl, "Self focusing of 2D arrays for SHM of plate-like structures using time reversal operator," in Structural Health Monitoring Condition-Based Maintenance and Intelligent Structures, F.-K. Chang, ed., p. 1119 27, DEStech Publications, Inc., cop, (Stanford, USA), September 1315 2011.

10. S. Doctor, T. Hall, and L. Reid, "SAFT the evolution of a signal processing technology for ultrasonic testing," NDT International 19(3), pp. 163 - 167, 1986.

11. L. Moreau, B. Drinkwater, and P. Wilcox, "Ultrasonic imaging algorithms with limited transmission cycles for rapid nondestructive evaluation," Ultrasonics, Ferroelectrics and Frequency Control, IEEE Transactions on 56, pp. 1932 -1944, september 2009.

12. M. Engholm and T. Stepinski, "Direction of arrival estimation of lamb waves using circular arrays," Structural Health Monitoring, 2010.

13. L. Liu and F. G. Yuan, "A linear mapping technique for dispersion removal of lamb waves," Structural Health Monitoring 9, pp. 75-86, January 2010.

14. P. Wilcox, "A rapid signal processing technique to remove the effect of dispersion from guided wave signals," Ultrasonics, Ferroelectrics and Frequency Control, IEEE Transactions on 50(4), pp. 419 -427, 2003.

15. B. Xu and V. Giurgiutiu, "Single mode tuning effects on Lamb wave time reversal withpiezoelectric wafer active sensors for structural health monitoring," Journal of Nondestructive Evaluation 26, pp. 123-134, 2007.

16. L. Ambrozinski, T. Stepinski, and T. Uhl, "Experimental comparison of 2D arrays topologies for SHM of planar structures," in Nondestructive Characterization for Composite Materials, Aerospace Engineering, Civil Infrastructure, and Homeland Security 2012, SPIE 8347, SPIE, July 3-6 2012. 